\title{
Limiar de VFC e diferentes métodos de identificação durante teste incremental máximo de corrida
}

http://dx.doi.org/10.11606/1807-5509201800030309

\author{
Eduardo Marcel Fernandes NASCIMENTO* \\ Maria Augusta Peduti Dal Molin KISS ${ }^{* *}$ \\ Flávio de Oliveira PIRES*
}

\author{
${ }^{*}$ Escola de \\ Artes, Ciências \\ e Humanidades, \\ Universidade de São \\ Paulo, São Paulo, SP \\ Brasil. \\ **Escola de Educação \\ Física e Esporte, \\ Universidade de São \\ Paulo, São Paulo, SP \\ Brasil.
}

\section{Resumo}

0 propósito do presente estudo foi obter evidências de validade e reprodutibilidade das respostas da variabilidade da frequência cardiaca (VFC) durante a corrida. Dezenove homens foram submetidos a dois testes progressivos máximos (teste e reteste com intervalos de 3 a 7 dias) com velocidade inicial $5 \mathrm{~km} . \mathrm{h}^{1}$ e incrementos de $1 \mathrm{~km} \cdot \mathrm{h}^{-1}$ a cada 3 minutos (1\% de inclinação constante) até exaustão. Trocas gasosas, lactato sanguineo e VFC foram obtidos durante todos os testes. Os resultados demonstraram que analises da VFC fornece boa estimativa dos limiares aeróbio e anaeróbio identificados pelas respostas ventilatórias e de lactato. Adicionalmente, os limiares de VFC demonstraram reprodutibilidade. Resultados confirmaram que os limiares aeróbio e anaeróbio podem ser identificados pela análise de um conjunto de dados da VFC.

PalAVRAS-CHAVE: Limiar aeróbio; Limiar anaeróbio; Regulação autonômica; Lactato sanguíneo; Consumo de oxigênio.

\section{Introdução}

Diferentes métodos têm sido utilizados para a análise da variabilidade da frequência cardíaca (VFC) durante exercício físico, tais como os que utilizam o domínio do tempo, o domínio da frequência e os métodos não lineares. Apesar dos questionamentos em relação a inadequada utilização de métodos no domínio da frequência para análise da VFC, uma vez que os dados obtidos durante o exercício apresentam, frequentemente, característica não-estacionária ${ }^{1}$, alguns estudos têm demonstrado a possibilidade do seu uso, sobretudo em testes incrementais máximos (TIM) ${ }^{2-4}$. Neste caso, as análises no domínio do tempo ${ }^{5}$, a plotagem de Poincaré e a entropia aproximada (ApEn) têm sido sugeridos como uma alternativa para a compreensão dos fatores randômicos e de previsibilidade na dinâmica dos intervalos R-R, quando os dados obtidos durante o exercício possuem característica não-estacionária, como no caso do TIM ${ }^{6}$.

Resultados encontrados em diferentes estudos reforçam a possibilidade da utilização da VFC para estimativa dos limiares, aeróbio (LAe) e anaeróbio (LAn), comumente identificados pela curva do lactato ou variáveis ventilatórias durante TIM ${ }^{2,3,6}$. Por exemplo, Үамамото et al. ${ }^{7}$ verificaram que a atividade do sistema nervoso parassimpático (SNP) diminuía de forma progressiva até intensidades próximas ao primeiro limiar ventilatório $\left(\mathrm{LV}^{1}\right)$, porém com um incremento gradativo na atividade do sistema nervoso simpático (SNS) em intensidades a partir de $\mathrm{LV}^{15,8}$.

A utilização da plotagem de Poincaré trouxe novas perspectivas para o uso da VFC em TIM, pois tornou possível a identificação de LAe e LAn a partir destes dados. Por exemplo, com relação ao LAe, Lima e KIss ${ }^{8}$ sugeriram a utilização do valor fixo de $3 \mathrm{~ms}$ em $\mathrm{SD}^{1}$ (desvio padrão da variabilidade instantânea, batimento a batimento) para a estimativa do primeiro limiar de lactato $\left(\mathrm{LL}_{1}\right)$, identificado na menor relação lactato sanguíneo [La]-carga de trabalho9. Posteriormente, KARAPETIAN 
et al. ${ }^{5}$ propuseram o uso do desvio padrão $(\mathrm{SD})$ e da média das diferenças sucessivas (MDS) de intervalos $\mathrm{R}-\mathrm{R}$ consecutivos, para a identificação de $\mathrm{LV}_{1}$ e $\mathrm{LL}_{1}$.

Com relação ao LAn, BuchHeIT et al. ${ }^{4}$ também sugeriram a utilização da VFC para a estimativa desta intensidade. Estudos de Cotrin et al. ${ }^{3,10}$ demonstraram que a análise da VFC no domínio da frequência identificava o segundo limiar ventilatório $\left(\mathrm{LV}_{2}\right) \mathrm{em}$ ciclistas e jogadores de futebol profissional, enquanto QUINART et al. ${ }^{11}$ observaram que métodos no domínio do tempo (raiz quadrada média das diferenças sucessivas de intervalos R-R consecutivos), e da frequência (transformada de Fourier) estavam correlacionados $(\mathrm{r}=0,66$ a 0,93$)$ ao $\mathrm{LV}_{2}$ em adolescentes treinados.

No entanto, apesar da sugestáo do uso dos dados de VFC para a determinaçáo de $\mathrm{LV}_{1}$ e $\mathrm{LL}_{1}$, assim como de $\mathrm{LV}_{2}$ e $\mathrm{LL}_{2}$, nenhum estudo verificou a similaridade entre estes pontos e as intensidades determinadas pelos limiares de VFC. A lacuna existente entre estes pontos ainda é motivo de debate na literatura, dada a aplicabilidade destes índices no diagnóstico clínico do nível de aptidão aeróbia ou na prescrição de treinamento físico ${ }^{12}$. Desde os trabalhos de GaEsSER and Poole ${ }^{13}$, estudos têm demonstrado diferentes resultados quando comparam $\mathrm{LL}_{1}$ e $\mathrm{LL}_{2}$ com $\mathrm{LV}_{1} \mathrm{e}$ $\mathrm{LV}_{2}$, respectivamente ${ }^{14-16}$. Consequentemente, um maior conhecimento sobre a utilização dos limiares de VFC na identificação destes limiares fisiológicos pode ser elucidativo. A confiabilidade e reprodutibilidade do método da VFC ainda estão por ser estabelecida.

Portanto, o objetivo do presente estudo foi verificar a possibilidade de estimativa de $\mathrm{LL}_{1} \mathrm{e}$ $\mathrm{LV}_{1}$, assim como de $\mathrm{LL}_{2}$ e $\mathrm{LV}_{2}$, por meio da VFC, durante TIM em esteira rolante. A reprodutibilidade dos dados foi verificada, a fim de se assegurar a fidedignidade dos resultados e métodos envolvidos.

\section{Método}

\section{Amostra}

Dezenove homens, praticantes de corrida (ao menos três vezes por semana), com idade de 30,4 $\pm 4,1$ anos, massa corporal de $74,3 \pm 8,5 \mathrm{~kg}$, estatura $\mathrm{de}^{17} 5,9 \pm$ $6,4 \mathrm{~cm}$ e percentual de gordura de 13,8 $\pm 4,6 \%$, participaram desse estudo. Eles eram saudáveis, isentos de tratamentos farmacológicos, não fumantes, e livres de qualquer tipo de distúrbio neuromuscular ou cardiovascular. Os participantes receberam explicaçóes verbais sobre riscos e benefícios do estudo e assinaram um consentimento informado antes da participação no mesmo. Os voluntários foram orientados a evitar atividades físicas extenuantes, consumo de refeições com alto conteúdo de gorduras ou proteínas, ingestão de bebidas contendo cafeína ou álcool, nas $24 \mathrm{~h}$ antecedentes aos testes. Ambos os TIM (teste e reteste) foram conduzidos sob condiçóes de laboratório (umidade relativa do ar $50 \pm 2 \%$ e temperatura $22 \pm$ $1^{\circ} \mathrm{C}$ ), com um intervalo de 3 e 7 dias entre eles. Os procedimentos adotados foram aprovados pelo Comitê de Ética da Escola de Educação Física e Esporte da Universidade de São Paulo ( $n^{\circ}$ 0010.0.342.000-10).

\section{Teste Incremental Máximo (TIM)}

Após preparação dos indivíduos aos procedimentos experimentais, foi iniciada aquisição das medidas

de frequência cardíaca (FC) e trocas gasosas por 10 minutos, enquanto os indivíduos repousavam numa maca. Ao final do período de repouso, uma amostra de $25 \mu l$ de sangue capilarizado foi coletada no dedo anelar dos indivíduos, e posteriormente armazenada a $-20^{\circ} \mathrm{C}$ para análise em analisador eletroenzimático (YSI 1500 - Yellow Springs Instruments - Ohio, EUA). Imediatamente após a coleta de sangue, os indivíduos foram conduzidos à esteira rolante (Imbramed, Super ATL) para início do TIM. Após aquecimento padrão de 3 minutos em velocidade de $5 \mathrm{~km} . \mathrm{h}-1$, o TIM foi iniciado a $5 \mathrm{~km} . \mathrm{h}-1$, com incrementos de $1,0 \mathrm{~km} . \mathrm{h}-1$ a cada três minutos, até a exaustão. Amostras de $25 \mu$ l de sangue arterializado foram coletadas durante os 30s finais de cada estágio. A esteira foi mantida com $1 \%$ de inclinação até o final do teste (protocolo adaptado de Heck et al. ${ }^{17}$ ).

As trocas gasosas, tais como ventilação (VE), volume de oxigênio consumido $\left(\mathrm{VO}_{2}\right)$ e dióxido de carbono produzido $\left(\mathrm{VCO}_{2}\right)$, foram medidas respiração-a-respiração, durante todo o teste (K4b2, COSMED ${ }^{\circledR}$. A calibração do sensor fluxo e dos analisadores de $\mathrm{O}_{2}$ (sensor de zircônio) e $\mathrm{CO}_{2}$ (absorção de infravermelho) foi realizada por meio de uma seringa contendo 3 litros de ar e cilindros com gases de composição conhecida, respectivamente.

Os dados da FC foram coletados batimentoa-batimento por cardiofrequencímetro S810i da $\operatorname{Polar}^{\oplus 18}$, com transmissão automática para 
computador, e posterior armazenagem em programa Polar Precision Performance 4.0. A velocidade (Vel) máxima atingida no TIM incremental foi registrada.

\section{Análise dos dados}

\section{Determinação do $\mathrm{VO}_{2 \mathrm{MÁx}}$}

Após conversão dos valores respiração a respiração para médias de $20 \mathrm{~s}$, o $\mathrm{VO}_{2 \mathrm{MÁx}}$ foi determinado de acordo com o critério proposto por Taylor et al. ${ }^{19}$, um platô na curva do $\mathrm{VO}_{2}$ identificado por um aumento no $\mathrm{VO}_{2}$ menor que $2,1 \mathrm{ml} \cdot \mathrm{kg}^{-1} \cdot \mathrm{min}^{-1}$, mediante incremento da intensidade. $\mathrm{Na}$ impossibilidade de determinação de platô de $\mathrm{VO}_{2}$, os seguintes critérios foram adicionalmente utilizados: 1) alcance da $\mathrm{FC}_{\text {MÁx }}$ predita pela idade (220-idade); 2) alcance de valores de $\left.[\mathrm{La}]>8,0 \mathrm{mmol} . \mathrm{l}^{-1} ; 3\right)$ razão de trocas respiratórias $(\mathrm{RER})>1,10^{20}$.

\section{Identificaçáo dos limiares de lactato}

Para identificação do $\mathrm{LL}_{1}$, foi utilizada a velocidade correspondente à [La] fixa de $2 \mathrm{mmol} . \mathrm{l}^{-121}$. Já para identificação do $\mathrm{LL}_{2}$ utilizou-se a concentração fixa de $3.5 \mathrm{mmol}^{-1} \mathrm{I}^{-1}$ de lactato, seguindo os critérios sugeridos por Нeck et al. ${ }^{17}$. Os limiares de lactato $\left(\mathrm{LL}_{1}\right.$ e $\left.\mathrm{LL}_{2}\right)$ foram expressos na velocidade correspondente, em valores absolutos Vel $\left(\mathrm{km} . \mathrm{h}^{-1}\right)$ e relativos $\left(\operatorname{Vel}_{\text {MÁX }} \%\right)$.

\section{Identificação dos limiares ventilatórios}

Após conversão dos dados das variáveis respiratórias para médias de 20s, três avaliadores experientes identificaram os limiares ventilatórios por meio de inspeção visual, da seguinte forma. A identificação do $L V$ foi realizada mediante o ponto de menor valor do equivalente de $\mathrm{O}_{2}\left(\mathrm{VE} / \mathrm{VO}_{2}\right)$, plotado em função do tempo, sem aumento concomitante do equivalente de $\mathrm{VCO}_{2}{ }^{12}$. Já para o $\mathrm{LV}_{2}$ foi utilizado o menor valor do equivalente de $\mathrm{VCO}_{2}\left(\mathrm{VE} / \mathrm{VCO}_{2}\right)$, antes de aumento progressivo. Caso os dados dos $\mathrm{VE} / \mathrm{VO}_{2}$ e $\mathrm{VE} / \mathrm{VCO}_{2}$ não apresentassem nitidez satisfatória, as fraçóes expiradas de oxigênio e dióxido foram utilizadas em associação, respectivamente ${ }^{12}$. Por fornecer valores menos susceptíveis a valores extremos, a mediana entre os avaliadores foi utilizada para expressar a intensidade correspondente a esses pontos $^{22}$. Valores de $\mathrm{LV}_{1} \mathrm{LV}_{2}$ foram expressos na velocidade correspondente, em termos absolutos $\left(\mathrm{km} \cdot \mathrm{h}^{-1}\right)$ e relativos $\left(\% \mathrm{Vel}_{\mathrm{MAX}}\right)$.

\section{Identificaçáo dos limiares da variabilidade da frequência cardíaca}

Após inspeção visual para verificar a qualidade dos dados de FC, os mesmos foram filtrados para eliminação de possíveis valores aberrantes de intervalos RR. Em seguida, os dados de intervalos R-R foram analisados por meio de plotagem de Poincaré, índices $\mathrm{SD}^{1}$ e desvio-padrão da variabilidade a longo prazo de intervalos $\mathrm{R}-\mathrm{R}$ contínuos $\left(\mathrm{SD}^{2}\right)$, em função de cada estágio. Os intervalos R-R do último minuto do repouso e do último minuto de cada estágio do TIM foram usados para identificação de dois limiares de VFC, descritos a seguir.

Primeiro modelo: A identificação do ponto onde ocorre uma significativa retirada vagal, normalmente coincidente com LV ${ }^{1}$ e LL ${ }^{1}$, foi obtida após Plotagem de Poincaré, por meio da variável $\mathrm{SD}^{18}$. Assim, a primeira carga $(\mathrm{km} . \mathrm{h}-1)$ correspondente aos valores de $\mathrm{SD}^{1} \leq 3 \mathrm{~ms}$ foi considerada como a intensidade referente ao $\mathrm{LV}^{1}$ e $\mathrm{LL}^{1}$, e chamado de $L V F C F^{1}$. Um segundo limiar de VFC, onde ocorre uma intensificação na participação do SNS, foi identificado $\left(\mathrm{LVFCF}^{2}\right)$ como sendo o ponto de menor valor encontrado na variável $\mathrm{SD}^{1}$.

Segundo modelo: A análise visual dos valores de VFC, feita por três avaliadores experientes e conhecedores do fenômeno, foi utilizada para identificação dos LVFC. Valores de SD1 e SD2 foram normalizados pela divisão da média do último minuto de cada estágio, pela média total dos intervalos RR durante todo o teste, e multiplicados por 100023,24. Após plotagem dos dados de VFC em função da intensidade, dois pontos de quebra de linearidade (decréscimo) em SD1 e SD2 identificaram $\mathrm{LL}^{1}$ e LV1 (LVFCN $\left.{ }^{1}\right)$, e $\mathrm{LL}^{2}$ e $\mathrm{LV}^{2}$ $\left(\mathrm{LVFCN}^{2}\right)$, respectivamente. A mediana entre os avaliadores foi utilizada para expressar a intensidade correspondente a esses pontos. A FIGURA 1 exemplifica a aplicação desse modelo visual.

Os limiares de VFC identificados pelo primeiro modelo (LVFC F ${ }^{1}$ e LVCF F ${ }^{2}$ ) e segundo modelo $\left(\mathrm{LVFCN}^{1} \mathrm{e} \mathrm{LVCFN}^{2}\right.$ ) foram expressos em valores absolutos $\left(\mathrm{km} \cdot \mathrm{h}^{-1}\right)$ e relativos (\%VelMÁX) da velocidade correspondente. 


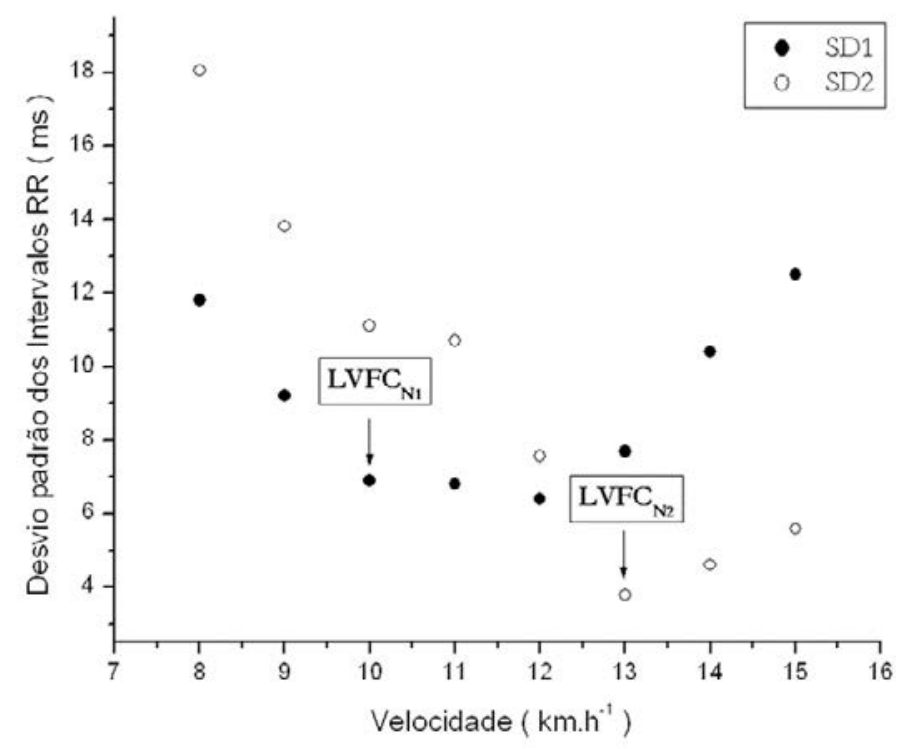

FIGURA 1 - Representação gráfica da determinação do limiar aeróbio e anaeróbio, por meio do segundo modelo proposto para identificação do primeiro limiar $\left(\mathrm{LVFC}_{\mathrm{N} 1}\right)$ e segundo limiar de variabilidade da frequência cardíaca $\left(\mathrm{LVFC}_{\mathrm{N}_{2}}\right)$, respectivamente. Desvio-padrão da variabilidade instantânea, batimento a batimento ( $\left.\mathrm{SD}^{1}\right)$ e desvio-padrão da variabilidade a longo prazo de intervalos $\mathrm{R}-\mathrm{R}$ contínuos $\left(\mathrm{SD}^{2}\right)$.

\section{Análise estatística}

A normalidade dos dados foi verificada e assegurada pelo teste de Shapiro-Wilk. Para análise descritiva utilizou-se média \pm desvio-padrão (DP). A ANOVA para medidas repetidas, acompanhada por post hoc de Bonferroni, verificou possíveis diferenças entre as intensidades determinadas pelos limiares de VFC (matemático e visual), e os demais limiares fisiológicos $\left(L_{1}\right.$ e LL $L_{2}$, e LV e LV $\left._{2}\right)$. O coeficiente de correlação de Pearson foi usado para verificar a associação entre os diferentes limiares. Adicionalmente, análise de Bland e Altman ${ }^{25}$ e coeficiente de correlação intraclasse (CCI) foram utilizados para analisar a reprodutibilidade dos limiares de VFC. Resultados foram interpretados como significantes quando $\mathrm{p}<0,05$.

\section{Resultados}

Quando teste e reteste foram comparados, $\mathrm{Vel}_{\mathrm{MÁX}}$ $\left(16.5 \pm 1.8 \mathrm{~km} \cdot \mathrm{h}^{-1} ; 16.6 \pm 1.7 \mathrm{~km} \cdot \mathrm{h}^{-1}\right), \mathrm{HR}_{\mathrm{MÁX}}$ $(192 \pm 6 \mathrm{bpm} ; 191 \pm 6 \mathrm{bpm}), \mathrm{VO}_{2 \text { MÁX }}(49.8 \pm 6.4$ $\left.\mathrm{ml} \cdot \mathrm{kg}^{-1} \cdot \mathrm{min}^{-1} ; 51.0 \pm 7.6 \mathrm{ml} \cdot \mathrm{kg}^{-1} \cdot \mathrm{min}^{-1}\right)$ e pico de [La] $\left(9.2 \pm 1.9 \mathrm{mmol} \cdot \mathrm{L}^{-1} ; 9.2 \pm 1.9 \mathrm{mmol} \cdot \mathrm{L}^{-1}\right)$ não apresentaram diferenças entre os testes $(p>0.05)$.

\section{Similaridade entre os diferentes limiares fisiológicos}

A TABELA 1 mostra os resultados referentes a todos os métodos utilizados para identificação dos diferentes limiares expressos em valores absolutos e relativos. Foram encontradas diferenças significantes $(\mathrm{p}<0,05)$ entre o $\mathrm{LVFCF}^{1}$ e os demais métodos de identificação do LAe (LL1, LV1 e LVFCN ${ }^{1}$ ), tanto em valores absolutos $\left(\mathrm{km} . \mathrm{h}-{ }^{1}\right)$ quanto relativos. Em relação ao LAn, não foram encontradas diferenças significativas entre os métodos $\left(\mathrm{LL}^{2}, \mathrm{LV}^{2}, \mathrm{LVFCF}^{2}\right.$ e $\mathrm{LVFCN}^{2}$ ), tanto em valores absolutos $\left(\mathrm{km} . \mathrm{h}^{-1}\right)$ quanto relativos $(\mathrm{p}>0,05)$.

A TABELA 2 apresenta os valores de coeficiente de correlação de Pearson entre os diferentes métodos de identificação do LAe e LAn, analisados em valores absolutos e relativos de Vel 
(km.h-1), respectivamente. O primeiro modelo de identificação dos limiares de VFC (LVFCF) apresentou níveis de correlação de $\mathrm{r}=0,60$ $(\mathrm{p}<0,01)$ e $\mathrm{r}=0,51(\mathrm{p}<0,05)$, quando comparados o LVFCF2 com o $\mathrm{LL}^{2}$ e $\mathrm{LV}^{2}$, respectivamente. O segundo modelo de identificação dos limiares pela VFC (LVFCN) apresentou valores maiores de correlaçáo tanto quando comparados o $\mathrm{LVFCN}^{1}$ $(\mathrm{r}=0,71$ e $\mathrm{r}=0,57)$ com os $\mathrm{LL}^{1}$ e $\mathrm{LV}^{1}(\mathrm{p}<0,01)$, respectivamente, assim como, quando comparados o $\operatorname{LVFCN}^{2}(\mathrm{r}=0,86$ e $\mathrm{r}=0,72)$ com os $\mathrm{LL}^{2}$ e $\mathrm{LV}^{2}$ $(\mathrm{p}<0,01)$, respectivamente.

TABELA 1 - Média e desvio-padrão referentes aos diferentes métodos de identificação do Limiar Aeróbio (A) e do Limiar Anaeróbio (B), expressos em valores absolutos e relativos da variável velocidade.

\begin{tabular}{lcc}
\hline & Valores Absolutos & Valores Relativos \\
\hline$(\mathbf{A})$ & $\mathbf{( k m \cdot \mathbf { h } ^ { - 1 } )}$ & \%Vel $_{\mathbf{M A x}}\left(\mathbf{k m} \cdot \mathbf{h}^{-1}\right)$ \\
\hline $\mathrm{LL}_{1}$ & $11,9 \pm 2,9^{\epsilon}$ & $71,4 \pm 11,1^{\epsilon}$ \\
$\mathrm{LV}_{1}$ & $11,3 \pm 1,8^{\epsilon}$ & $68,6 \pm 7,4^{\epsilon}$ \\
$\mathrm{LVFC}_{\mathrm{F} 1}$ & $9,0 \pm 1,9$ & $55,0 \pm 9,0$ \\
$\mathrm{LVFC}_{\mathrm{N} 1}$ & $11,6+1,6^{€}$ & $70,6 \pm 6,3^{€}$ \\
\hline$(\mathbf{B})$ & & \\
\hline $\mathrm{LL}_{2}$ & $14,2 \pm 2,5$ & $85,7 \pm 7,3$ \\
$\mathrm{LV}_{2}$ & $14,2 \pm 1,5$ & $86,4 \pm 4,7$ \\
$\mathrm{LVFC}_{\mathrm{F} 2}$ & $12,8 \pm 2,6$ & $77,4 \pm 10,7$ \\
$\mathrm{LVFC}_{\mathrm{N} 2}$ & $14,2 \pm 2,0$ & $86,1 \pm 6,3$ \\
\hline
\end{tabular}

TABELA 2 - Valores de coeficientes de correlação de Pearson entre os diferentes métodos de identificação do limiar aeróbio e anaeróbio, expressos em valores absolutos de velocidade (km.h-1) na situação teste.

\begin{tabular}{lcccc}
\hline & $\mathbf{L}$ & $\mathbf{L V}$ & $\mathbf{L V F C}_{\mathbf{F}}$ & $\mathbf{L V F C}_{\mathbf{N}}$ \\
\hline $\mathrm{LL}$ & 1 & $0,58^{* *}$ & 0,39 & $0,71^{* *}$ \\
$\mathrm{LV}$ & $0,86^{* *}$ & 1 & 0,28 & $0,57^{* *}$ \\
$\mathrm{LVFC}_{\mathrm{F}}$ & $0,60^{* *}$ & $0,51^{*}$ & 1 & $0,61^{* *}$ \\
$\mathrm{LVFC}_{\mathrm{N}}$ & $0,86^{* *}$ & $0,72^{* *}$ & $0,55^{*}$ & 1 \\
\hline
\end{tabular}

\section{Reprodutibilidade dos diferentes limiares fisiológicos}

Os resultados dos níveis de CCI encontrados nas situaçóes teste e reteste quanto aos diferentes métodos de identificação de LAe e LAn, são mostrados na TABELA 3 sendo expressos em valores absolutos para a variável Vel $\left(\mathrm{km} \cdot \mathrm{h}^{-1}\right)$. $\mathrm{O}$ primeiro modelo utilizado para identificação do $\mathrm{LAn}\left(\mathrm{LVFC}_{\mathrm{F} 2}\right)$ apresentou valores de $\mathrm{r}=0,69$ $(\mathrm{p}<0,001)$. Já para o segundo modelo utilizado para identificação do LAe $\left(\operatorname{LVFC}_{\mathrm{N} 1}\right)$ e LAn
$\left(\mathrm{LVFC}_{\mathrm{N} 2}\right)$, apresentaram valores de $\mathrm{r}=0,80$ e $\mathrm{r}=$ $0,79(\mathrm{p}<0,001)$, respectivamente.

A FIGURA 2 mostra os resultados da plotagem de Bland-Altman, referentes aos métodos $\mathrm{LVFCF}^{2}$, $\mathrm{LVFCN}^{1}$ e LVFCN ${ }^{2}$, respectivamente, sendo os valores expressos quanto a variável Vel (km.h-1). Os valores médios do LVFCF2 ficaram em torno de $-0,20$ representados pela linha média, com um $\mathrm{DP} \pm 3 \mathrm{Vel}\left(\mathrm{km}^{\mathrm{h}} \mathrm{h}^{1}\right) \cdot \mathrm{O} \mathrm{LVFCN}^{1}$ e o $\mathrm{LVFCN}^{2}$ apresentaram valores de linha média em torno de $-0,15$ e $-0,20$, respectivamente, assim como, valores médios de $\mathrm{DP} \pm 2 \mathrm{Vel}\left(\mathrm{km} \cdot \mathrm{h}^{-}{ }^{-}\right)$em ambos.
$€$ diferenças significativas em relação ao LVFCF1 $(p<0,05)$.
Triângulo superior direito coeficientes de correlação referentes aos valores do Limiar Aeróbio

Triângulo inferior esquerdo coeficientes de correlação referentes aos valores do Limiar Anaeróbio ${ }^{*} e^{* *}$ coeficientes de correlação significativos estatisticamente em um nível $p<0,05$ e $p<0,01$, respectivamente. 
TABELA 3 - Valores absolutos referentes aos níveis de coeficiente de correlação intraclasse, expressos nos diferentes métodos de identificação dos limiares metabólicos, em relação à variável velocidade $\left(\mathrm{km} . \mathrm{h}^{-1}\right)$.

** estatisticamente significante para um $\mathrm{p}$ $<0,001$;

$\begin{array}{cc}\text { LL1 } & 0,89^{* *} \\ \text { LL2 } & 0,93^{* *} \\ \text { LV1 } & 0,72^{* *} \\ \text { LV2 } & 0,75^{* *} \\ \text { LVFCF1 } & 0,79^{* *} \\ \text { LVFCF2 } & 0,69^{* *} \\ \text { LVFCN1 } & 0,80^{* *} \\ \text { LVFCN2 } & 0,79^{* *}\end{array}$

A
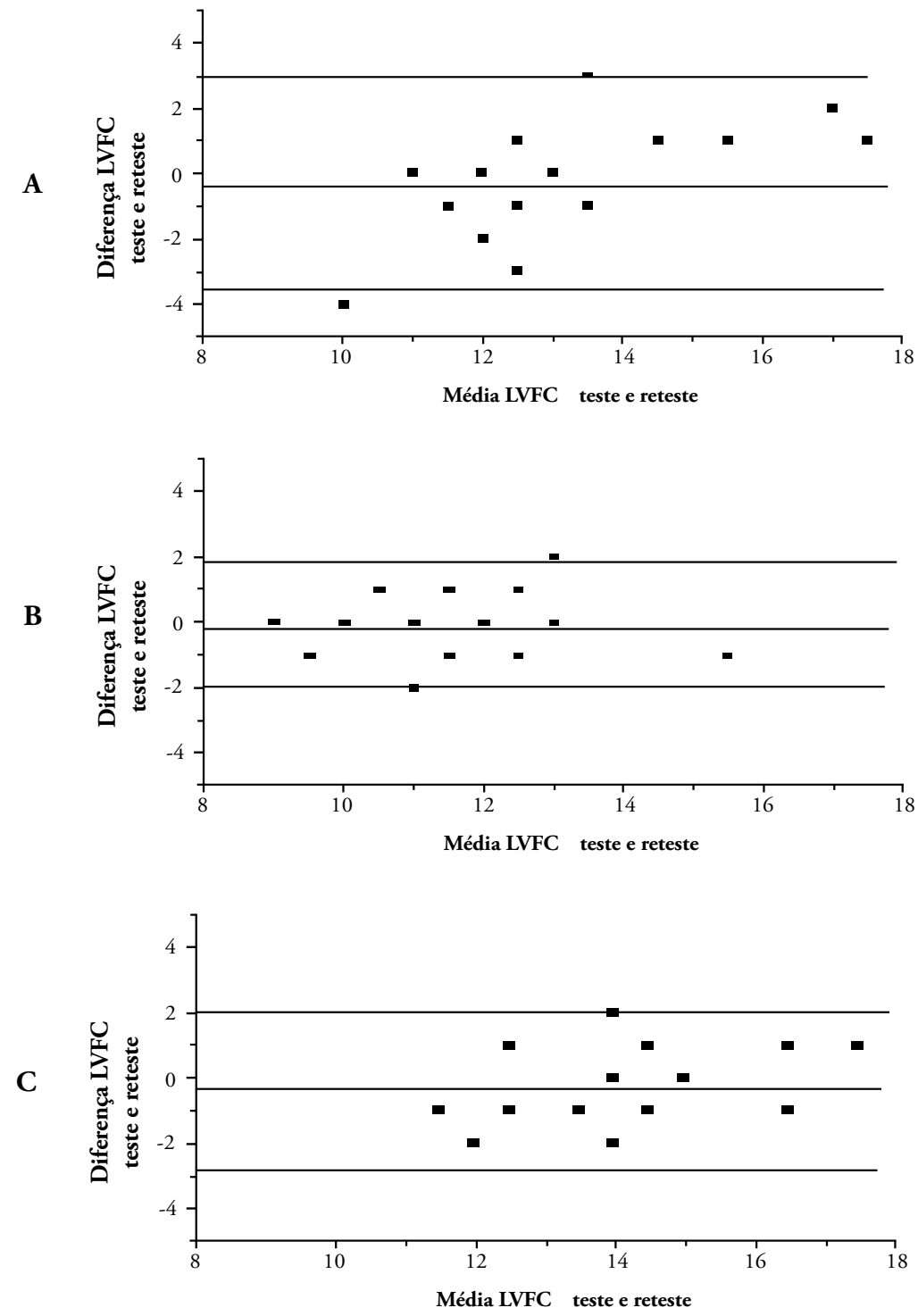

FIGURA 2 - Representação gráfica dos valores identificados pela análise de Bland-Altman, referentes à identificação do segundo limiar de variabilidade da frequência cardíaca (LVFCF2), proposto pelo primeiro modelo (A), e identificação do primeiro limiar (LVFCN1) e segundo limiar (LVFCN2) de variabilidade da frequência cardíaca, propostos pelo segundo modelo (B e C, respectivamente), por meio da variável Vel (km.h-1). 


\section{Discussão}

Os principais resultados do presente estudo foram: a) a possibilidade de determinaçáo do LAe e LAn por meio dos LVFC, em valores absolutos e relativos de Vel $\left(\mathrm{km} \cdot \mathrm{h}^{-1}\right)$, FC (bpm) e $\mathrm{VO}_{2}\left(\mathrm{ml} \cdot \mathrm{kg}^{-1}\right.$. min..$\left.^{-1}\right)$; b) positiva reprodutibilidade referente aos modelos de identificação do LAe e LAn.

Em relaçáo ao LAe, os resultados encontrados indicam que o $\mathrm{LVFCF}^{1}$ foi diferente do $\mathrm{LL}^{1}$ e $\mathrm{LV}^{1}$ $(\mathrm{p}<0,05)$, embora LVFCN1 tenha provido valores próximos aos $\mathrm{LL}^{1} \mathrm{e} \mathrm{LV}$, quando expressos em valores absolutos ou relativos ao $\mathrm{VO}^{2}$ (ml.kg-1.min.-1), a FC (bpm) e a Vel (km.h-1). No presente estudo, $\mathrm{LVFCF}^{1}$ foi identificado de acordo com o método proposto por Lima e KIss ${ }^{8}$. Esses autores analisaram o comportamento da VFC durante TIM em cicloergômetro e identificaram um platô de decréscimo em SD ${ }^{1}$ (3ms), demonstrando não haver diferenças entre a intensidade de $\mathrm{LL}^{1}$, detectado pela menor relação [La]/carga, e a intensidade identificada pelo LVFC estabelecido pelo valor fixo de $3 \mathrm{~ms} \mathrm{em} \mathrm{SD}^{1}$. Os autores sugeriram a utilização do platô de $3 \mathrm{~ms}$ como uma alternativa para a estimativa de $\mathrm{LL}^{1}$, indicando a transição entre a significativa retirada vagal e o aumento da participação autonômica simpática.

Posteriormente, NAKAMURA et al. ${ }^{26}$ utilizaram a mesma metodologia proposta por Lima e KIss ${ }^{8}$ para analisar os efeitos do treinamento aeróbio sobre o LVFC. Os autores observaram que o LVFC foi sensível aos efeitos do treinamento aeróbio de curto prazo, reforçando a ideia de que este possa ser indicador da capacidade aeróbia. Tal sugestão encontra suporte na provável redução da participação simpática, e maior modulação parassimpática, em intensidades moderadas de exercício. Estudos prévios demonstraram que, durante TIM em cicloergômetro, existe uma aproximação entre a retirada vagal e a primeira zona de transição metabólica, permitindo a sua identificação pelo comportamento da VFC, trocas gasosas ${ }^{6,7}$ e lactato sanguíneo ${ }^{5}$. Todavia, é importante ressaltar que alteraçóes no ergômetro, magnitude de incremento (intensidade e duração) e condiçóes ambientais podem afetar a resposta destas variáveis e, consequentemente, interferir na intensidade demarcada por elas. Por exemplo, a VFC pode apresentar diferentes comportamentos entre testes em cicloergômetro e esteira rolante, uma vez que a transiçáo entre caminhada e corrida (6,0 a 7,5 km.h-1) pode ocasionar modificaçáo da eficiência metabólica ${ }^{27-30}$, alterando o controle autonômico cardiovascular.
Por outro lado, não foram encontradas diferenças ( $p$ > 0,05) entre LVFCN ${ }^{1}$ e LL ${ }^{1}$ ou LV ${ }^{1}$. Quando expressos em termos relativos, foram encontrados valores em torno de 70\% e 69\% da VelMÁX, para os $\mathrm{LVFCF}^{1}$ e $\mathrm{LVFCN}^{1}$, respectivamente, valores próximos à primeira zona de transição metabólica ${ }^{31}$. Isso corrobora os achados de KARAPETIAN et al. ${ }^{5}$, que indicaram a possibilidade de uso da VFC para determinar a primeira zona de transição metabólica. Os autores verificaram que o limiar de VFC apresentava boa concordância com os demais métodos.

Com relação à segunda zona de transição metabólica, nossos resultados revelam não haver diferenças em relação aos demais métodos utilizados para detectá-la. Ademais, correlação significante $(r=0,86 ; \mathrm{p}<0,01)$ foi observada entre LVFCN ${ }^{2} \mathrm{eLL}^{2}$, e entre $\mathrm{LVFCF}^{2} \mathrm{e} \mathrm{LL}^{2}$ $(r=0,60 ; p<0,01)$, apesar da correlaçáo moderada neste segundo. Estudos prévios corroboram similaridade e correlação para variáveis cardiorrespiratórias em diferentes tipos de teste $\mathrm{e}^{3,4,32}$.

Lima e $\mathrm{KIss}^{8}$ propuseram a possibilidade de ocorrência de um segundo ponto de estabilização na variável SD $\mathrm{SD}^{1}$ em torno de $80 \%$ da carga de pico em cicloergômetro, e especularam que este ponto poderia estar associado ao LAN. ABAD et al. ${ }^{33}$, aplicando metodologia semelhante com onze jogadores de futebol da categoria juvenil, adotando como critério a primeira carga na qual a média fosse inferior a $2 \mathrm{~ms}$, identificaram um segundo ponto de estabilização na variável $\mathrm{SD}^{1}$ (L2VFC), com valores expressos em torno de 78\% da $\operatorname{Vel}_{\text {MÁX }}\left(\mathrm{km} \cdot \mathrm{h}^{-1}\right)$. Os autores sugeriram a utilização do L2VFC como um possível método alternativo de identificação do LAn. Todavia, os resultados do estudo de ABAD et al..$^{33}$ devem ser vistos com cautela, haja vista que os autores não reportaram valores de limiares identificados por metodologia padrão, tal como $\mathrm{LL}_{2}$ ou $\mathrm{LV}_{2}$.

Um dos pontos importantes desse estudo é a análise da reprodutibilidade dos limiares, sobretudo os que utilizaram a resposta da VFC. Os resultados encontrados no presente estudo indicam bons níveis de CCI $(0,69$ a 0,$89 ; \mathrm{p}<0,001)$ em relação aos LVFC. Adicionalmente, os valores do CCI para $\mathrm{LL}^{1}$ e $\mathrm{LL}^{2}$ foram 0,89 e 0,93 , respectivamente $(p<0,01)$. Já para os níveis de CCI relacionados aos $\mathrm{LV}^{1} \mathrm{e} \mathrm{LV}^{2}$ foram 0,72 e $0,75(p<0,01)$, respectivamente. Além disso, plotagem de Bland-Altman demonstrou haver uma boa similaridade para os métodos $\mathrm{LVFF}^{2}, \mathrm{LVFCN}^{1}$ e LVFCN2, entre as situaçóes teste e reteste, uma vez que os valores se encontraram dentro 
do intervalo específico de confiança estipulado. Coletivamente, esses dados indicam que os métodos que utilizaram a VFC na determinação de limiares fisiológicos tradicionais são reprodutivos, desde que seja utilizada a padronizaçáo dos procedimentos empregada neste estudo.

Do ponto de vista aplicado, este estudo fornece um novo modelo com diferentes métodos de identificação do LAe e LAn pela analise da VFC, sendo estes de relativa simplicidade em sua utilização e que requer um custo mais acessível em comparação aos métodos tradicionais.

Resultados da presente investigação sugerem a possibilidade de identificação de LAe por meio da VFC, mediante utilização do $\mathrm{LVFC}_{\mathrm{N} 1}$. Em adição, ambos os $\mathrm{LVFC}_{\mathrm{F} 2}$ e $\mathrm{LVFC}_{\mathrm{N} 2}$ parecem prover razoáveis estimativas do Lan. Ao menos para TIM em esteira, com procedimentos similares aos empregados na presente investigação, os métodos de VFC analisados apresentam bons níveis de reprodutibilidade.

\section{Abstract}

HRV threshold and different methods of identification during an incremental exhaustive running test

The purpose of this study was to obtain evidence of validity and reproducibility of the heart rate variability (HRV) response during running. Nineteen men performed two maximum progressive tests (test and retest with intervals of 3 to 7 days) with an initial speed of $5 \mathrm{~km} \cdot \mathrm{h}^{-1}$ and increments of $1 \mathrm{~km} \cdot \mathrm{h}^{-1}$ every 3 minutes (1\% constant slope) until exhaustion. Gas exchange, blood lactate and heart rate variability data were obtained throughout the tests. Results showed that analysis of the HRV provided good estimates of aerobic and anaerobic thresholds identified by lactate and ventilation responses. Additionally, HRV thresholds provided reproducible thresholds. Results confirmed that aerobic and anaerobic thresholds may be identified by HRV data set.

KEYWORDS: Aerobic threshold; Anaerobic threshold; Autonomic regulation; Blood lactate; Oxygen uptake.

\section{Referências}

1. Casadei B, Cochrane S, Johnston J, Conway J, Sleight P. Pitfalls in the interpretation of spectral analysis of the heart rate variability during exercise in humans. Acta Physiol Scand. 1995;153(2):125-31.

2. Cottin F, Médigue C, Leprête PM, Papelier Y, Koralsztein, JP, Billat V. Heart rate variability during exercise performed below and above ventilatory threshold. Med Sci Sports Exerc. 2004;36(4):594-600.

3. Cottin F, Médigue C, Lopes P, Leprêtre PM, Heubert R, Billat V. Ventilatory thresholds assessment from heart rate variability during an incremental exhaustive running test. Int J Sports Med. 2007;28(4):287-94.

4. Buchheit M, Solano R, Millet GP. Heart-rate deflection point and the second heart-rate variability threshold during exercise in trained boys. Pediatr Exerc Sci. 2007;19(2):192-204.

5. Karapetian GK, Engels HJ, Gretebeck RJ. Use of heart rate variability to estimate LT and VT. Int J Sports Med. 2008;29(8):652-7.

6. Tulppo MP, Mäkikallio TH, Takala TE, Seppänen T, Huikuri HV. Quantitative beat-to-beat analysis of heart rate dynamics during exercise. Am J Physiol. 1996;271(1 Pt 2):H244-52.

7. Yamamoto Y, Hughson RL, Nakamura Y. Autonomic nervous system responses to exercise in relation to ventilatory threshold. Chest. 1992;101(5):206S-210S.

8. Lima JRP, Kiss MAPDM. Limiar de variabilidade da frequência cardíaca. Rev Bras Ativ Fís Saúde. 1999;04(1):29-38.

9. Berg A, Jakob E, Lehmann M, Dickhuth HH, Huber G, Keul J. Aktuelle Aspekte der modernen Ergometrie. Pneumologie. 1990;44(1):2-13.

10. Cottin F, Leprête PM, Lopes P, Papelier Y, Médigue C, Billat V. Assessment of ventilatory thresholds from heart rate variability in well-trained subjects during cycling. Int J Sports Med. 2006;27(12):959-67.

11. Quinart S, Mourot L, Nègre V, Simon-Rigaud ML, Nicolet-Guénat M, Bertrand AM, et al. Ventilatory thresholds determined from HRV: comparison of 2 methods in obese adolescents. Int J Sports Med. 2014;35(3):203-8. 
12. Meyer T, Lucía A, Earnest CP, Kindermann W. A conceptual framework for performance diagnosis and training prescription from submaximal gas exchange parameters - theory and application. Int J Sports Med. 2005;26(1):S38-48.

13. Gaesser GA, Poole DC. Lactate and ventilatory thresholds: disparity in time course of adaptations to training. J Appl Physiol. 1986;61(3):999-1004.

14. Leti T, Mendelson M, Laplaud D, Flore P. Prediction of maximal lactate steady state in runners with an incremental test on the field. J Sports Sci. 2012;30(6):609-16.

15. Racinais S, Buchheit M, Girard O. Breakpoints in ventilation, cerebral and muscle oxygenation, and muscle activity during an incremental cycling exercise. Front Physiol. 2014;5:142.

16. Ribeiro J, Figueiredo P, Sousa M, De Jesus K, Keskinen K, Vilas-Boas JP, Fernandes RJ. Metabolic and ventilatory thresholds assessment in front crawl swimming. J Sports Med Phys Fitness. 2015;55(7-8):701-7.

17. Heck H, Mader A, Hess G, Mücke S, Müller R, Hollmann W. Justification of the 4-mmol/l lactate threshold. Int J Sports Med. 1985;6(3):117-30.

18. Gamelin FX, Berthoin S, Bosquet L. Validity of the polar S 810 heart rate monitor to measure R-R intervals at rest. Med Sci Sports Exerc. 2006;38(5):887-93.

19. Taylor HL, Buskirk E, Henschel A. Maximal oxygen intake as an objective measure of cardiorespiratory performance. J Appl Physiol. 1955;8(1):73-80.

20. Howley ET, Bassett DR Jr, Welch HG. Criteria for maximal oxygen uptake: review and commentary. Med Sci Sports Exerc. 1995;27(9):1292-301.

21. Kindermann W, Simon G, Keul J. The significance of the aerobic-anaerobic transition for the determination of work load intensities during endurance training. Eur J Appl Physiol Occup Physiol. 1979;42(1):25-34.

22. Pires FO, Silva AEL, Gagliardi JFL, Barros RV, Degaki E, Kiss MAPD. Possibilidade de ocorrência dos $1^{\circ}$ e $2^{\circ}$ limiares ventilatórios em cargas de trabalho coincidentes, durante protocolos progressivos com incrementos de longa duração. R Bras Ci e Mov. 2005;13(1):61-9.

23. Mourot L, Bouhaddi M, Perrey S, Rouillon JD, Regnard J. Quantitative Poincaré plot analysis of heart rate variability: effect of endurance training. Eur J Appl Physiol. 2004;91(1):79-87.

24. Tulppo MP, Mäkikallio TH, Seppänen T, Laukkanen RT, Huikuri HV. Vagal modulation of heart rate during exercise: effects of age and physical fitness. Am J Physiol. 1998;274(2, Pt 2):H424-9.

25. Bland JM, Altman DG. Statistical methods for assessing agreement between two methods of clinical measurement. Lancet. 1986;1(8476):307-10.

26. Nakamura FY, Aguiar CA, Fronchetti L, Aguiar AF, Lima JRP. Alteração do limiar de variabilidade da frequência cardíaca após treinamento aeróbio de curto prazo. Motriz. 2005;11(1):1-9.

27. Monteiro WD, Araujo CGS. Transição caminhada-corrida: considerações fisiológicas e perspectivas para estudos futuros. Rev Bras Med Esporte. 2001;7(6):207-22.

28. Patla AE, Sparrow WA. Factors that have shaped human locomotor structure and behavior: the 'joules' in the crown. In: Sparrow WA, editor. Energetics of human activity. Champaign: Humans Kinetics; 2000. p. 43-65.

29. Nascimento EMF, Silva AEL, Bertuzzi RCM, Kiss MAPDM, Pires FO. Caracterização da curva da frequência cardíaca durante teste incremental máximo em esteira. Rev Bras Cineantropom Desempenho Hum [Internet]. 2011 [citado 1 abr 2019];13(4):285-91. Disponível: https://bit.ly/2UmItq1

30. Boullosa DA, Barros ES, del Rosso S, Nakamura FY, Leicht AS. Reliability of heart rate measures during walking before and after running maximal efforts. Int J Sports Med. 2014;35(12):999-1005.

31. Skinner JS, McLellan TM. The transition from aerobic to anaerobic metabolism. Res Q Exerc Sport. 1980;51(1):234-48.

32. Buchheit M, Al Haddad H, Millet GP, Lepretre PM, Newton M, Ahmaidi S. Cardiorespiratory and cardiac autonomic responses to 30-15 intermittent fitness test in team sport players. J Strength Cond Res. 2009;23(1):93-100.

33. Abad CCC, Barros RV, Oliveira FR, Lima JRP, Pereira B, Kiss MAPM. O segundo platô da variabilidade da frequência cardíaca indica o segundo limiar de transição fisiológica? Efdeportes [Internet]. 2007 [citado 1 abr 2019]:12(114). Disponível: https://bit.ly/2TQDFER.

ENDEREÇO

Eduardo Marcel Fernandes Nascimento Rua Isabel de Castela, 478, ap. 23 - Vila Madalena 05445-010 - São Paulo - SP - BRASIL E-mail: eduardomarcel@usp.br
Recebido para publicação: 01/10/2015

1a. Revisão: 29/06/2016

Aceito: 28/07/2016 